

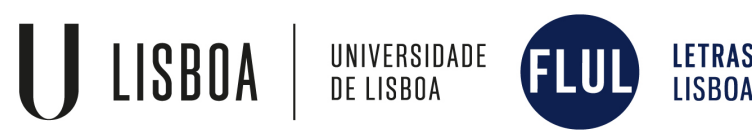

OPHIUSSA. Revista do Centro de Arqueologia da Universidade de Lisboa

ISSN 1645-653X / E-ISSN 2184-173X

Publicação anual

Volume $2-2018$

Direcção e Coordenação Editorial:

Ana Catarina Sousa

Elisa Sousa

Conselho Científico:

André Teixeira (Universidade Nova de Lisboa)

Carlos Fabião (Universidade de Lisboa)

Catarina Viegas (Universidade de Lisboa)

Gloria Mora (Universidad Autónoma de Madrid)

Grégor Marchand (Centre National de la Recherche Scientifique)

João Pedro Bernardes (Universidade do Algarve)

José Remesal (Universidade de Barcelona)

Leonor Rocha (Universidade de Évora)

Manuela Martins (Universidade do Minho)

Maria Barroso Gonçalves (Instituto Superior de Ciências do Trabalho e da Empresa)

Mariana Diniz (Universidade de Lisboa)

Raquel Vilaça (Universidade de Coimbra)

Xavier Terradas Battle (Consejo Superior de Investigaciones Científicas)

Secretariado: André Pereira

Capa: André Pereira sobre vaso cerâmico de Camposoto (desenho de António Sáez Romero / Joan Ramon Torres).

Paginação: Elisa Sousa

Impressão: Europress

Data de impressão: Dezembro de 2018

Edição impressa (preto e branco): 300 exemplares

Edição digital (a cores): www.ophiussa.letras.ulisboa.pt

ISSN: 1645-653X / E-ISSN 2184-173X

Depósito legal: 190404/03

Copyright @ 2018 , os autores

Edição:

UNIARQ - Centro de Arqueologia da Universidade de Lisboa, Faculdade de Letras de Lisboa, 1600-214 - Lisboa.

www.uniarq.net - www.ophiussa.letras.ulisboa.pt - uniarq@letras.ulisboa.pt

Revista fundada por Victor S. Gonçalves (1996).

O cumprimento do acordo ortográfico de 1990 foi opção de cada autor.

Esta publicação é financiada por fundos nacionais através da FCT - Fundação para a Ciência e a Tecnologia, I.P., no âmbito do projecto UID/ARQ/00698/2013. 


\section{EQUIPAMENTOS DE CULTO NOS SANTUÁRIOS DA IDADE DO FERRO DO SUL DE PORTUGAL: OS ALTARES}

\section{CULTIC FEATURES IN THE SOUTHERN PORTUGUESE IRON AGE SANCTUARIES: THE ALTARS}

FRANCISCO B. GOMES'

\section{RESUMO}

A presença de um conjunto de áreas de combustão estruturadas interpretáveis como altares em diversos espaços de culto da Idade do Ferro do Sul de Portugal encontra-se bem atestada. A variedade de contextos em que estas estruturas se enquadram implica, contudo, uma considerável diversidade morfológica, construtiva e mesmo funcional que traduz a adaptação destas estruturas às práticas sociais e rituais das comunidades que as utilizaram.

Palavras-chave: Altares; Santuários; Idade do Ferro; Ritual; Comensalidade.

\section{ABSTRACT}

The presence of a series of structured combustion areas that can be interpreted as altars in several cult spaces of the southern Portuguese Iron Age is currently well attested. The variety of contexts in which these structures were found implies, however, a considerable morphologic, constructive and even functional diversity which translates their adaptation to the concrete social and ritual practices of the communities which used them.

Keywords: Altars; Sanctuaries; Iron Age; Ritual; Commensality. 


\section{«(...) E logo aprontaram para o deus \\ a sagrada hecatombe em torno do bem construído altar. \\ Lavaram as mãos e pegaram nos grãos de cevada.» llíada, I, 447-9 \\ (trad. de Frederico Lourenço) \\ 1. OS ALTARES DOS SANTUÁRIOS DA IDADE DO FERRO: OBSERVAÇÕES PRÉVIAS}

Num trabalho recente dedicado aos espaços religiosos da Idade do Ferro no Sul do actual território português tive a oportunidade de assinalar a existência recorrente nesses espaços de um conjunto de equipamentos arquitectónicos que se podem relacionar funcionalmente com as actividades próprias do culto (Gomes 2012: 134-7).

Entre esses equipamentos chama particularmente a atenção um conjunto de áreas de combustão estruturadas cuja interpretação funcional como altares pode, em muitos casos, sustentar-se não apenas pelas marcas de utilização que apresentam como, sobretudo, pela análise contextual sistemática dos ambientes onde se implantam (Fig. 1).

Este tipo de estruturas foram contudo documentadas em âmbitos muito diversificados entre si (Figs. 1 e 2). Pode com efeito resenhar-se, em primeiro lugar, a sua presença numa série de ambientes enquadráveis em contextos de matriz oriental ou "orientalizante", de que são exemplo os casos da Fase II de Abul A (Alcácer do Sal) (Mayet Silva 2000: 144 e 167-8), dos sucessivos santuários de Castro Marim (Arruda et al. 2009; Gomes 2012) ou mesmo do hipotético altar do Castro dos Ratinhos (Moura) (Berrocal-Rangel - Silva 2010; Prados Martínez 2010: 269), de difícil caracterização.

Essa presença é, de resto, paralelizável com a documentação proveniente de um conjunto já avultado de espaços de culto documentado em território espanhol e cuja importância na estruturação do território durante a I Idade do Ferro tem sido reiteradamente sublinhada (Celestino 1997; Belén 2000a; 2000b; 2001; Arruda - Celestino 2009).

A presença de altares em diversos desses espaços encontra-se bem atestada, merecendo destaque, neste contexto, as amplamente estudadas estruturas de Coría del Río (Escacena - Izquierdo 2000; Escacena 2002: 42-66), do Carambolo (Sevilha) (Fernández Flores - Rodríguez Azogue 2007) e de Cancho Roano (Badajoz) (Celestino 1994; 2008: 3301), bem como as mais recentemente documentadas na região de Málaga (Arancibia Román - Escalante Aguilar 2006: 338 e Lám. I; Escalante Aguilar et al. 2012; Sánchez et al. 2012: 80-1) e em Casas del Turuñuelo (Badajoz) (Rodríguez González - Celestino Pérez 2017).

A documentação do território português não se esgota, contudo, nestes contextos de clara influência exógena, havendo também a resenhar a presença de áreas de combustão estruturadas de tipo altar noutros sítios onde o influxo mediterrâneo parece ter sido mais difuso, predominando rasgos de matriz claramente local (Arruda 2001).

Entre estes contamos com documentos do tipo que aqui se pretende analisar quer no Baixo Alentejo, concretamente na área de Castro Verde - Neves II e Corvo I (Maia - Correa 1985; Maia - Maia 1986; Maia 1987; 1988; 2008) - quer mesmo já no Alentejo Central, no sítio de Espinhaço de Cão (Alandroal) (Calado - Mataloto 2008: 196; Mataloto 2009).

Evidentemente, esta diversidade de contextos traduz-se em distintas características morfológicas e construtivas, havendo também algumas evidências de possíveis usos diferenciados, dados dos quais me ocuparei nas páginas que se seguem.

\section{ASPECTOS CONSTRUTIVOS E MORFOLÓGICOS}

Do ponto de vista da técnica construtiva as várias estruturas de tipo altar documentadas podem distribuir-se fundamentalmente em três grandes

\begin{tabular}{|c|c|c|c|c|}
\hline Estrutura & Cronologia (a.n.e.) & Morfologia & Téc. Cons trutiva & Dim. (m) \\
\hline C. dos Ratinhos - F.1b & f. séc. IX - séc. VIII & Rectangular & Adobe & c. $1,6 \times 0,63$ \\
\hline Abul A - F.II & $2^{\mathrm{a}} \mathrm{m}$. séc. VII & Rectangular & Pedra + Pedra moída & $1,35 \times 1,25$ \\
\hline C. Marim - F.III & $2^{\mathrm{a}} \mathrm{m}$. séc. VII & Quadrangular (c/ cabeceira) & Pedra + Argila & $1 \times 1$ \\
\hline C. Marim - F.IV & séc. VI & Rectangular (c/ receptáculo) & Pedra + Argila & $0,92 \times 1,30$ \\
\hline C. Marim - F.V & $2^{\mathrm{a}} \mathrm{m}$. séc. V & Rectangular & Pedra + Argila & $0,88 \times 0,69$ \\
\hline Espinhaço de Cão & séc. VI & Quadrangular & Adobe & $0,7 \times 0,7$ \\
\hline Neves II & f. séc. VI - séc. V & Quadrangular & Pedra+Seixos & $1,32 \times 1,45$ \\
\hline Corvo I & $2^{\mathrm{a}} \mathrm{m}$. séc. V & Quadrangular & Pedra+Seixos & ND \\
\hline
\end{tabular}

Fig. 1 - Características gerais das áreas de combustão estruturadas de tipo "altar" referidas no texto. 
grupos. O primeiro, representado pelos exemplos de Castro Marim (Fases III, IV e V) (Arruda - Freitas Oliveira 2007: 471, 475; Arruda et al. 2009: 79-80) (Fig. 3) e de Abul A (Fase II) (Mayet - Silva 2000: 144, 167-8) (Fig. 4), caracteriza-se pela presença de uma moldura pétrea preenchida por argila ou, no caso de Abul A, pedra moída. O altar mais antigo de Castro Marim (Fase III) apresentava ainda a particularidade de incorporar fragmentos cerâmicos nesse enchimento central (Arruda - Freitas - Oliveira 2007: 475; Arruda et al. 2009: 79).

Um segundo grupo, circunscrito à área de Castro Verde (Fig. 5), caracteriza-se igualmente pela presença de uma moldura pétrea que aqui, contudo, envolvia uma área de combustão formada por seixos quartzíticos rolados argamassados com argila, que pelo menos no caso de Corvo I apresentava uma tendência circular (Maia - Correa 1985: 247-8; Maia Maia 1996: 87-8).

Finalmente, haveria a assinalar um terceiro grupo representado por estruturas de combustão realizadas em adobe, documentadas quer no compartimento interior do Castro dos Ratinhos (Prados Martínez 2010: 269) (Fig. 6) quer ainda no hipotético compartimento de culto do complexo de Espinhaço de Cão (Calado - Mataloto 2008: 196) (Fig. 7).

Já no que diz respeito à sua morfologia, a variabilidade destas estruturas é algo mais substancial, ainda que predominem de forma geral as configurações quadrangulares ou rectangulares. Assim, um primeiro grupo formado por estruturas de configuração quadrangular ou sub-quadrangular simples inclui os casos de Abul A, datável da segunda metade do século VII a.n.e., de Espinhaço de Cão (século VI a.n.e.), de Neves II (finais do século VIséculo $V$ a.n.e.), de Corvo I (segunda metade do século $V$ a.n.e.) e também da Fase $V$ de Castro Marim (também da segunda metade do século $V$ a.n.e.).

Quanto ao possível altar do santuário do Castro dos Ratinhos (final do século IX - início do VIII a.n.e.), a sua caracterização é, como se disse, difícil de precisar, mas parece ter assumido, também ele, uma morfologia grosso modo rectangular, sem que possa precisar-se se apresentava algum rasgo particular.

A difusão e perduração desta modalidade formal deverá ser interpretada como uma função directa da sua simplicidade morfológica que a torna particularmente fácil de adaptar a recursos e técnicas construtivas diversificadas bem como a usos e funções também eles distintos, como adiante terei oportunidade de comentar.

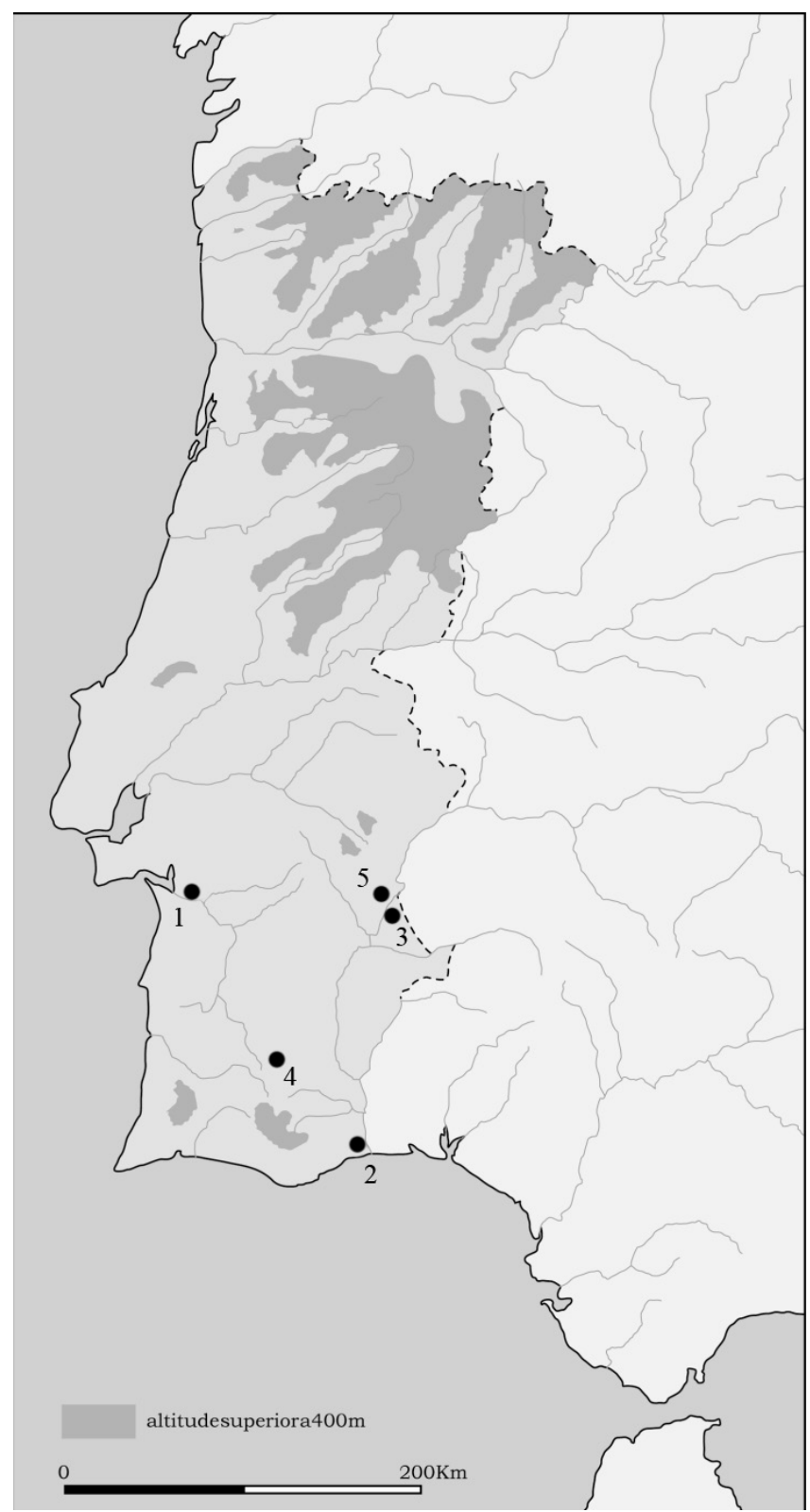

Fig. 2 - Distribuição dos sítios analisados no texto: 1. Abul A (Alcácer do Sal); 2. Castelo de Castro Marim; 3. Castro dos Ratinhos (Moura); 4. Sítios da área de Neves-Corvo (Castro Verde); 5. Espinhaço de Cão (Alandroal). Localização aproximada. Base cartográfica do Professor Doutor Victor S. Gonçalves.

De facto, as únicas estruturas que não se enquadram nesta morfologia de base são as dos santuários das fases mais antigas de Castro Marim que apresentam especificidades que os diferenciam dentro do conjunto em apreço. De facto, o altar da Fase III (segunda metade do século VII a.n.e.), embora de morfologia geral quadrangular, apresentava no seu lado Oeste a adição de uma plataforma sobrelevada composta por blocos pétreos formando 

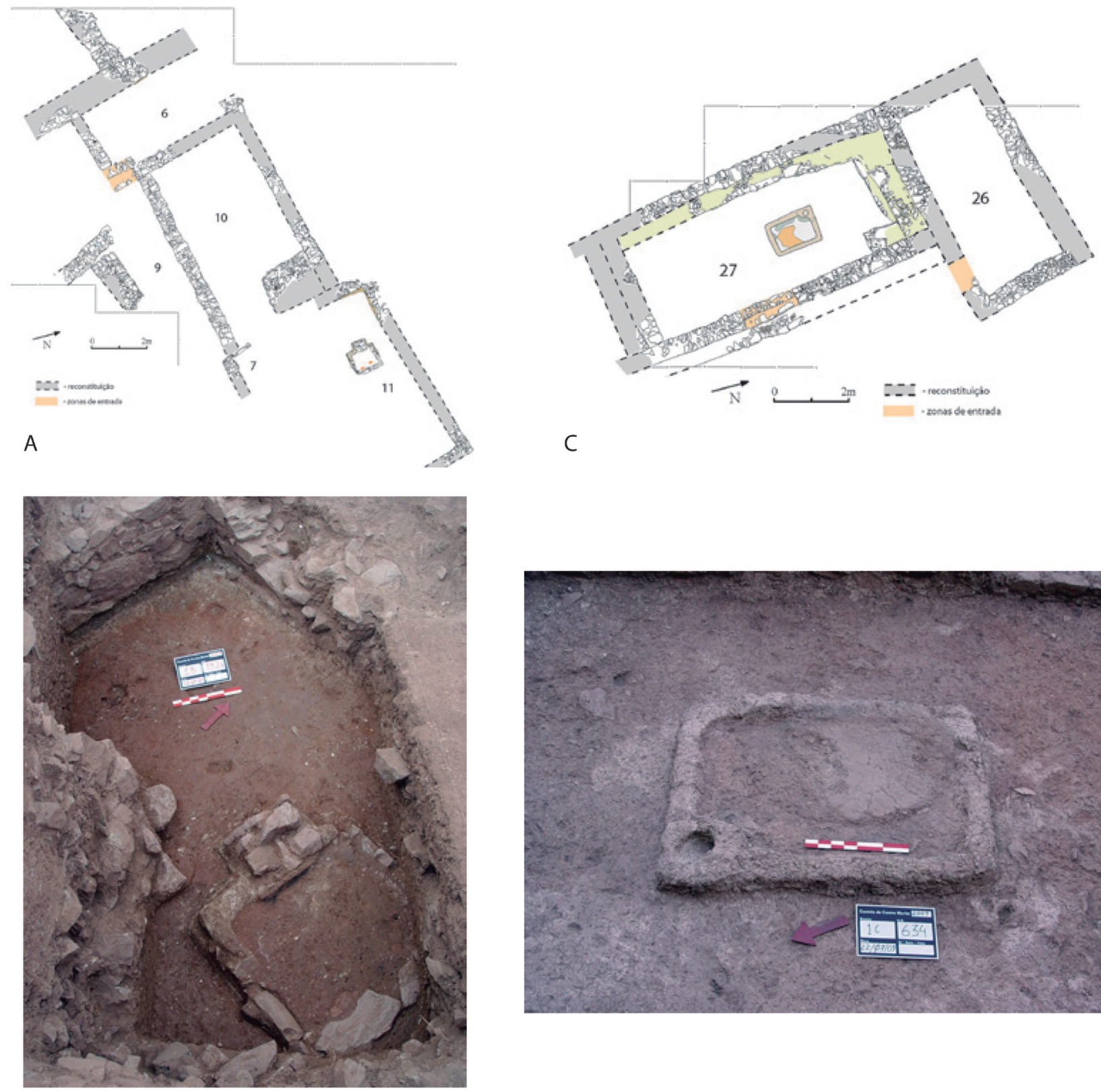

C

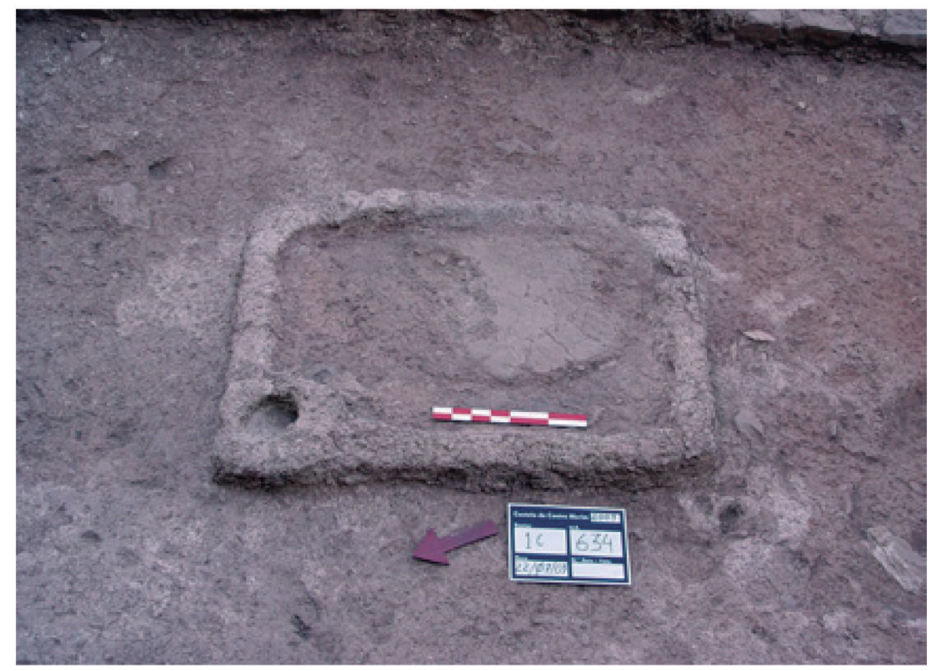

B

$\mathrm{D}$

Fig. 3 - Santuários e altares do Castelo de Castro Marim: A. Santuário da Fase III (seg. Arruda et al. 2009); B. Altar da Fase III (foto cedida pela Professora Doutora Ana Margarida Arruda); C. Santuário da Fase IV (seg. Arruda et al. 2009); D. Altar da Fase IV (foto cedida pela Professora Doutora Ana Margarida Arruda).

uma espécie de cabeceira (Arruda - Freitas - Oliveira 2007: 475; Arruda et al. 2009: 79), o que o diferencia das restantes estruturas conhecidas.

De igual modo, o altar da Fase IV (século VI a.n.e.), de tendência genericamente rectangular com cantos arredondados, diferenciava-se dos demais pelo facto de a sua moldura pétrea apresentar, no seu canto Noroeste, um alargamento configurando uma concavidade circular com cerca de $12 \mathrm{~cm}$ de diâmetro, peculiaridade igualmente única no panorama das estruturas deste tipo aqui recolhidas (Arruda - Freitas - Oliveira 2007: 471; Arruda et al. 2009: 80).

Ainda ao nível do comentário à morfologia destas estruturas parece-me pertinente salientar a inexistência, até ao momento, de qualquer exemplo enquadrável nos chamados altares em 
forma de pele de touro, ou de lingote cipriota. Este grupo formal, cuja origem oriental parece hoje plenamente demonstrada (Gómez Peña 2010; 2011; v. contudo Marín Ceballos 2006), encontra-se muito bem documentado no território espanhol e tem sido objecto de considerável atenção por parte da investigação recente (Celestino Pérez 1994; 2008; Escacena - Izquierdo 2000; Escacena 2002; Escacena - Coto 2010; Gómez Peña 2010; 2011; 2012; 2012-3; Almagro Gorbea et al. 2011-2), constituindo-se como um dos aspectos mais característicos dos ambientes de culto de matriz oriental ou "orientalizante".

De facto, do ponto de vista formal, a morfologia taurodérmica é largamente predominante no conjunto dos altares documentados tanto na Andaluzia como mesmo na Extremadura, pelo que a sua inexistência em território português não deixa de ser peculiar. No entanto caberia assinalar neste apartado a interpretação que ultimamente se tem feito dos chamados larnakes do sítio de Neves I (Castro Verde) (Maia 1987) (Fig. 8) como altares (Celestino Pérez 2008: 331-2; Escacena - Coto 2010: 159-160; Almagro Gorbea et al. 2011-2: 242).

Originalmente, e no quadro de uma leitura do sítio como necrópole, foi atribuída a estas peças uma função funerária enquanto contentores cinerários (Maia 1987: 240). Esta interpretação de Neves I foi já objecto de um escrutínio sistemático (Arruda 2001: 281-2; Gomes 2012: 74-78), parecendo hoje de descartar uma funcionalidade funerária para o sítio, em substituição da qual se propôs a sua leitura como espaço de culto.

Em trabalho anterior tive já, por outro lado, oportunidade de me pronunciar sobre a interpretação funcional dos larnakes, tendo então rejeitado, porventura precipitadamente, a sua interpretação como altares, embora salientando a sua associação a restos de combustão e a eventuais rituais de fogo (Gomes 2012: 137). Hoje, contudo, vejo como mais plausível essa hipótese alternativa, por distintas razões.

Por um lado, em Neves I não se documentou qualquer estrutura edificada de tipo altar. É verdade que os altares não são rigorosamente indispensáveis nos ambientes de culto, mas o certo é que no conjunto de santuários que tive oportunidade de analisar a vasta maioria dispunha de estruturas deste tipo, sendo porventura o sítio baixo-alentejano a excepção mais notável (Gomes 2012: Fig. 67).

Por outro lado, as evidências da utilização daquilo que poderíamos designar como altares móveis em ambientes orientais/"orientalizantes"
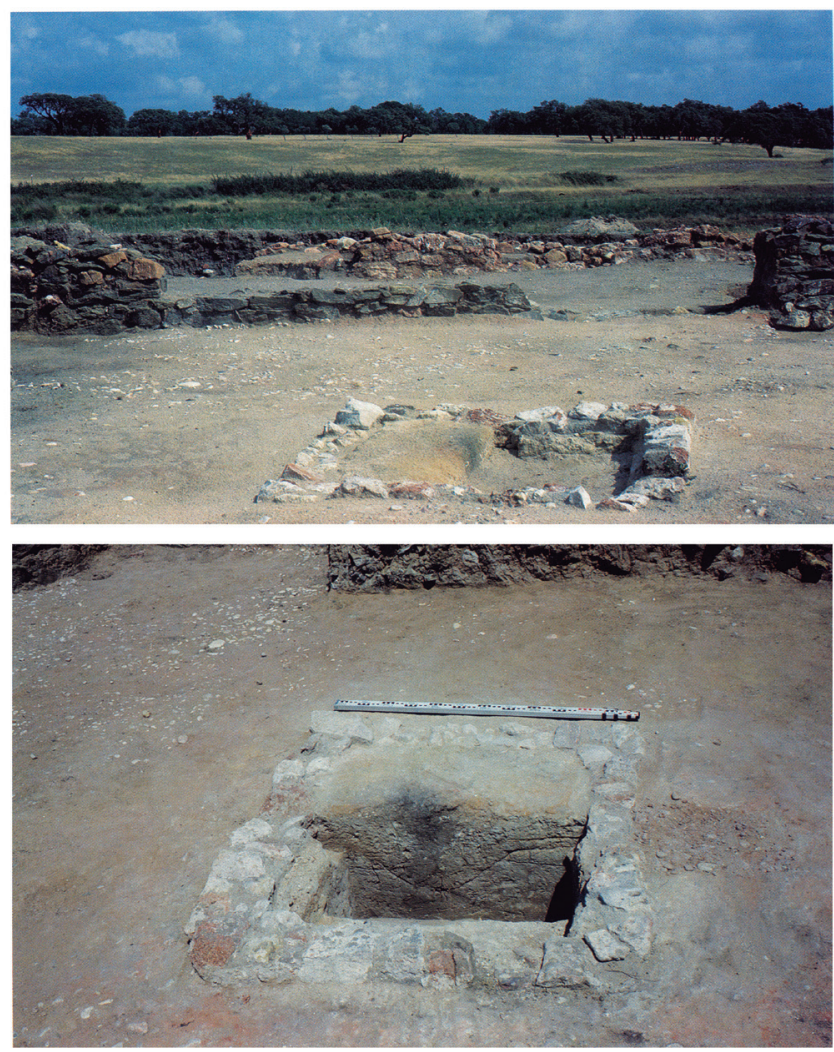

Fig. 4 - Vistas do altar da Fase II de Abul A (seg. Mayet - Silva 2000).

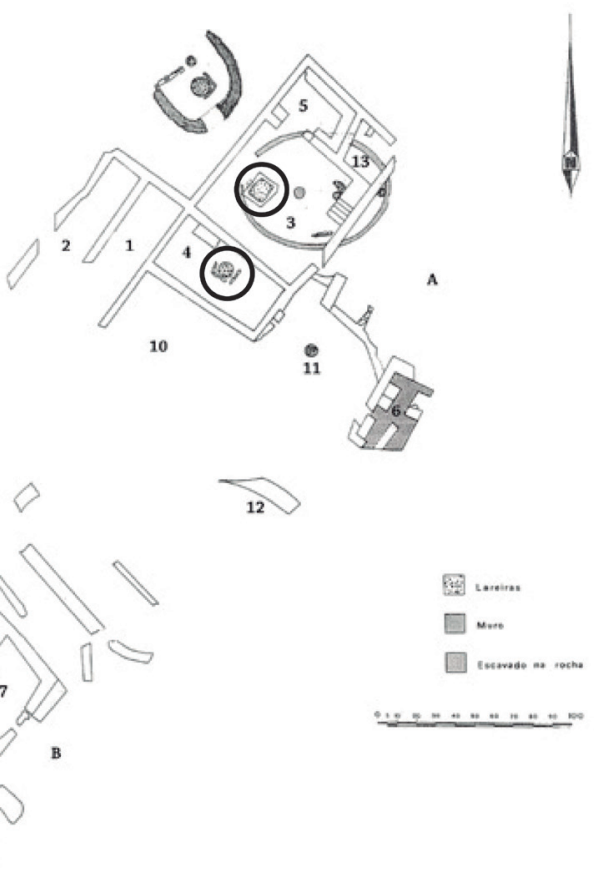

Fig. 5 - Complexo de Neves I (Castro Verde) (seg. Maia Maia 1986, adaptado). Os círculos assinalam as áreas de combustão estruturadas eventualmente associadas a funções rituais. 


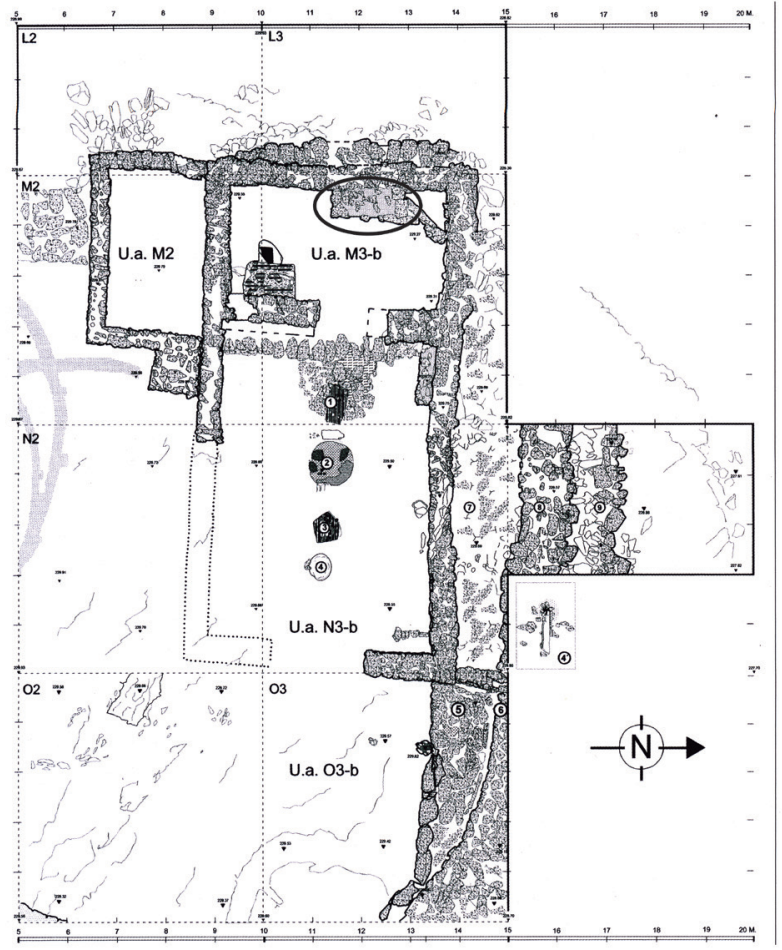

Fig. 6 - Santuário da Fase 1b do Castro dos Ratinhos (Moura) (seg. Berrocal-Rangel - Silva 2010, adaptado). O círculo assinala o possível altar do compartimento interior (M3).

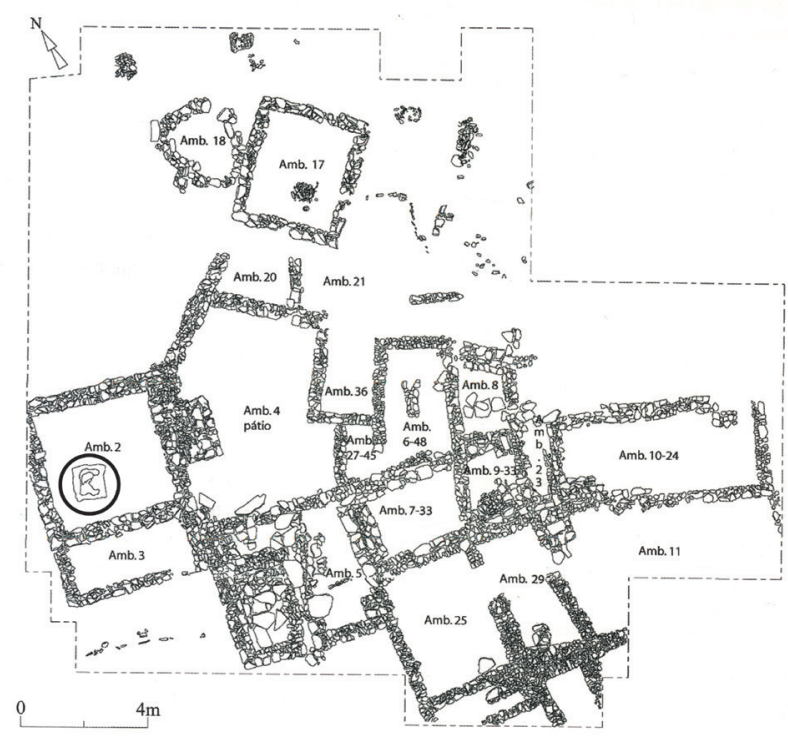

Fig. 7 - Planta do complexo de Espinhaço de Cão (Alandroal) (seg. Calado - Mataloto 2008, adaptado). O círculo assinala o possível altar do Ambiente 2. do Sul peninsular parece hoje, na esteira de um interessante trabalho de J. L. Escacena e Ma. Coto (2010), bastante bem documentada, algo que de resto se pode também assinalar para outros ambientes mediterrâneos (cf. Spagnoli 2012).

Finalmente, o próprio contexto das peças de Neves I poderia suportar uma interpretação deste tipo. A sua sobreposição num mesmo ponto no centro de um compartimento de características destacadas, por exemplo, parece-me sugestiva, recordando casos como os de Cancho Roano (Celestino Pérez 1994; 2001) ou de Coría del Río (Escacena - Izquierdo 2000) em que, embora os altares possam amortizar-se aquando de episódios de remodelação, a sua posição é posteriormente assinalada por novos altares que se Ihes sobrepõem de forma geralmente rigorosa.

Insisto, contudo, que na ausência de estudos concretos sobre os restos osteológicos que aparentemente se recuperaram associados a estas peças (Maia 1987: 240), no sentido de confirmar se se tratam de restos humanos ou faunísticos, a interpretação destas peças não pode dar-se como caso encerrado.

\section{USOS E FUNÇÕES}

A questão da utilização dada às estruturas antes listadas não é fácil de abordar. De facto, o registo arqueológico é frequentemente deficitário no que diz respeito à informação relativa àquilo que poderíamos designar o gesto ritual, o que necessariamente dificulta a restituição das práticas concretas associadas ao culto. Não obstante existem alguns dados de interesse que permitem sustentar que o panorama foi, também a este nível, algo diversificado.

Desde logo, uma interpretação global de todas estas estruturas como altares sacrificiais, destinados à prática de oferendas cruentas (Almagro Gorbea et al. 2011-2: 243), embora pertinente à luz das evidências disponíveis sobre a importância dos sacrifícios de animais no culto durante o período em estudo (Bandera Romero 2002), corre o risco de reduzir e ocultar outro tipo de práticas e de gestos que poderão ter sido tão ou mais importantes nalguns contextos concretos.

A única constante nos casos portugueses aqui analisados parece ser a presença de evidências de combustão as quais, contudo, se podem facilmente relacionar com distintas actividades.

Entre estas merece menção a eventual prática de oferendas não cruentas, com particular destaque 


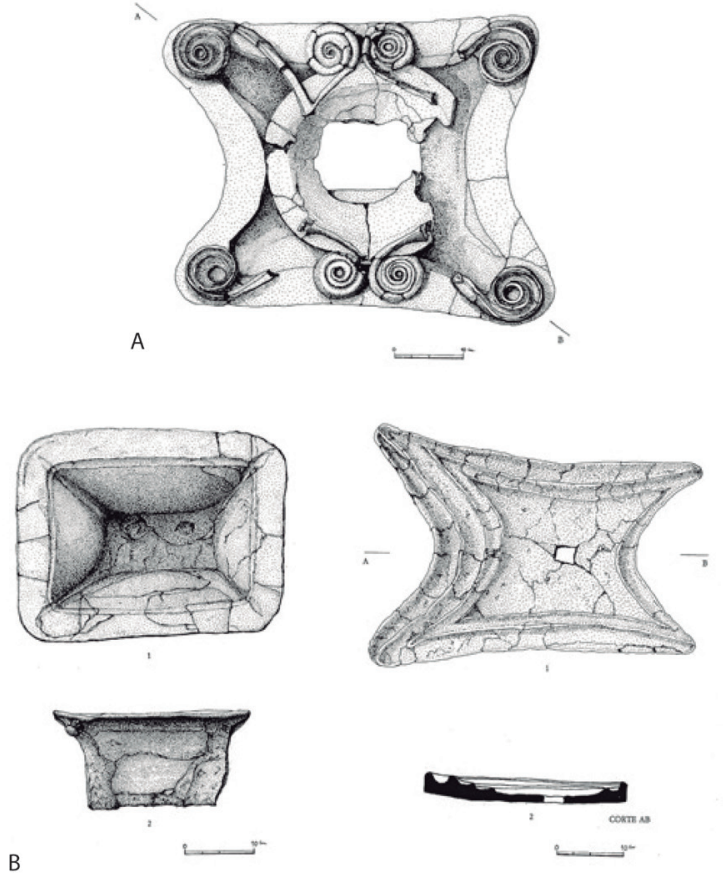

Fig. 8 - Larnakes de Neves II (seg. Maia 1987): A. Larnax B; B. Larnax A.

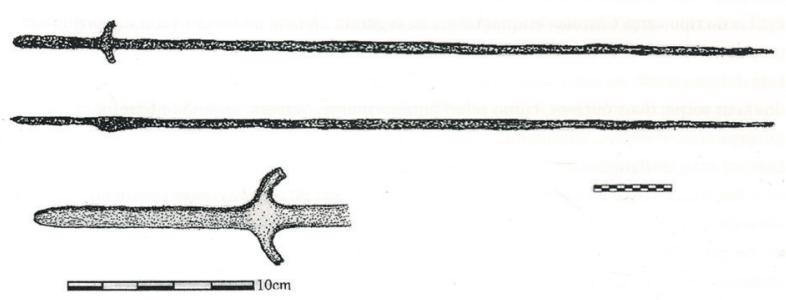

Fig. 9 - Espeto de ferro recolhido sobre o possível altar de Corvo 1 (seg. Maia 1986).

para a queima de essências aromáticas, interpretação que se propôs, por exemplo, para o altar de Abul A (Mayet - Silva 2000: 167-8). Devo salientar que a utilização de óleos e perfumes em ambientes rituais se encontra muito bem atestada quer nos contextos portugueses (Gomes 2012: 138; 2015) quer mais latamente nos âmbitos orientais e "orientalizantes" do Sul peninsular (López Rosendo 2005), o que confere uma particular verosimilitude à utilização dos altares para este fim, sem excluir a utilização, amplamente documentada, de outros equipamentos móveis, nomeadamente thymiateria metálicos e queimadores cerâmicos de várias tipologias (idem; cf. tb. Gomes 2015).
Por outro lado, e se é certo que a cabeceira do altar da Fase III de Castro Marim se poderia facilmente relacionar com uma funcionalidade sacrificial, o receptáculo que caracteriza o altar da Fase IV - e que pode aproximar-se funcionalmente (ainda que não morfologicamente) dos que se documentam nalguns dos altares de Coría del Río (Escacena - Izquierdo 2000; Escacena 2002), de Málaga (Arancibia Román Escalante Aguilar 2006: Lám. I) ou de Cancho Roano (Celestino Pérez 1994; 2001) - poderia igualmente associar-se com outro tipo de libações (de água, vinho ou óleos, por exemplo) que não necessariamente de natureza sanguinolenta (Almagro Gorbea et al. 20112: 242), o que por outro lado não exclui de modo algum a bem atestada prática da combustão.

Uma outra evidência muito sugestiva das eventuais utilizações destas estruturas foi documentada em Corvo I, onde se recuperou, sobre a estrutura de combustão antes comentada, um espeto de ferro (Fig. 9), aparentemente in situ (Maia 1986; Maia - Maia 1996: 87-8), que pela sua identidade morfológica com os bem conhecidos obeloi de bronze (Almagro Gorbea 1974; Gamito 1988) deverá relacionar-se com a preparação e o consumo de carne.

Esta evidência sugere a existência de práticas, muito provavelmente ritualizadas, de consumo de alimentos, que podemos suspeitar terem-se revestido de um carácter comunitário e colectivo. De facto, se a este elemento de juízo somarmos a abundante presença, em espaço aparentemente contíguo, de recipientes áticos para beber, nomeadamente de Taças "Cástulo", bem como de contentores anfóricos, aparentemente de importação (Maia Maia 1996: 88), podemos considerar que existem fortes evidências de que neste complexo se tenham realizado cerimónias de natureza simposiástica nas quais a referida estrutura de tipo altar serviria de foco e ponto de congregação.

Foi já de resto salientada a forte possibilidade de que a comensalidade constituísse um aspecto relevante na estruturação social destas comunidades sidéricas do interior alentejano (Gomes 2014: 38-9), o que justificaria plenamente a sua transferência para uma esfera eminentemente ritualizada.

Podemos assim suspeitar que as restantes estruturas de tipo altar documentadas em contextos do interior alentejano - caso de Neves II ou de Espinhaço de Cão - poderiam responder a lógicas de utilização semelhantes, sobretudo na medida em que os mesmos se enquadram em complexos de natureza aparentemente polifuncional onde a componente 
doméstica parece também bem evidenciada.

Nestes núcleos de natureza eminentemente rural as práticas de culto revestir-se-iam, com toda a probabilidade, de uma natureza eminentemente comunitária, estando provavelmente a cargo dos chefes das linhagens familiares que terão ocupado os numerosos polos de ocupação dispersa que se têm vindo a documentar (Arruda 2001; Mataloto 2004; 2009; Antunes et al. 2017), incorporando-se inevitavelmente nas estratégias de competição e afirmação social de cada comunidade num ambiente sociopolítico descentralizado e heterárquico (Gomes 2012: 144).

Pode assim defender-se que embora compartindo um carácter eminentemente vinculado ao domínio do culto, as estruturas aqui comentadas e que, como tive oportunidade de salientar, se distribuem por âmbitos distintos, desempenharam funções diferenciadas, em conformidade com as práticas sociais e rituais concretas das comunidades que os utilizaram e que em torno delas estruturaram pelo menos parte das suas práticas religiosas.

Faltam-nos ainda dados contextuais mais finos para avaliar das eventuais relações entre estas diversas práticas rituais - sacrifícios cruentos, não cruentos, queima de perfumes/óleos, práticas comensais - que, como anteriormente referido, não têm porque ser mutuamente exclusivas e podem constituir distintos aspectos dentro das práticas rituais de uma mesma comunidade, ou inclusivamente distintos passos em rituais específicos de natureza complexa.

Ainda assim, creio que ficou demonstrada a existência de casos concretos que remetem de forma preferencial para facetas específicas dentro deste pacote de práticas rituais. $\mathrm{O}$ reconhecimento desta diversidade de rituais afigura-se-me de resto como um passo importante para implementar, de futuro, metodologias e estratégias de análise que permitam uma aproximação mais sustentada ao gesto ritual das comunidades sidéricas do Sudoeste peninsular.

\section{AGRADECIMENTOS}

Gostaria de expressar a minha gratidão à Professora Doutora Ana Margarida Arruda pela cedência das fotografias de campo dos altares por si escavados e identificados no Castelo de Castro Marim, que se reproduzem na Figura 3.

Nota: o presente artigo não segue o Acordo Ortográfico da Língua Portuguesa de 1990.

\section{REFERÊNCIAS BIBLIOGRÁFICAS}

ALMAGRO GORBEA, M. (1974) - Los asadores de bronce del suroeste peninsular. Revista de Archivos, Bibliotecas y Museos 77: 351-395.

ALMAGRO GORBEA, M. - LORRIO ALVARADO, A. J. MEDEROS MARTÍN, A. - TORRES ORTIZ, M. (2011-2) El mito de Telepinu y el altar primordial en forma de piel de toro. Cuadernos de Prehistoria y Arqueología de la Universidad Autónoma de Madrid 37-38: 241-262.

ANTUNES, A.S. -DEUS, M. -ESTRELA, S. - LARRAZABAL, J. - SOARES, A. M. - SALVADOR MATEOS, R. (2017) Monte do Bolor 3, Monte do Pombal 2, Salsa 3 e Torre Velha 3: contextos de planície da I Idade do Ferro do Alentejo Interior. In JIMÉNEZ ÁVILA, J. (Coord.) Sidereum Ana III. El Río Guadiana y Tartessos. Mérida: 159-186.

ARANCIBIA ROMÁN, A. - ESCALANTE AGUILAR, M. a del M. (2006) - La Málaga Fenicio-Púnica a la luz de los últimos hallazgos. Mainake 28: 333-360.

ARRUDA, A. M. (2001) - A Idade do Ferro PósOrientalizante no Baixo Alentejo. Revista Portuguesa de Arqueologia 4-2: 207-291.

ARRUDA, A. M. - CARRETERO POBLETE, P. A. - FREITAS, V. T. de - SOUSA, E. de - BARGÃO, P. - LOURENÇO, P. OLIVEIRA, C. F. (2009) - Castro Marim: un santuário en la desembocadura del Guadiana. In MATEOS CRUZ, P. - CELESTINO PÉREZ, S. (eds.) - Santuarios, oppida y ciudades: arquitectura sacra en el origen y desarrollo urbano del Mediterráneo Occidental. Madrid: 79-88.

ARRUDA, A. M. - CELESTINO PÉREZ, S. (2009) Arquitectura Religiosa en Tartessos. In MATEOS CRUZ, P. - CELESTINO PÉREZ, S. (eds.) - Santuários, oppida y ciudades. Arquitectura religiosa en el origen y desarrollo urbano del Mediterráneo Occidental. Madrid: 29-77.

ARRUDA, A. M. - FREITAS, V. T. de - OLIVEIRA, C. F. (2007) - Os Fenícios e a urbanização no Extremo Ocidente: o caso de Castro Marim. In LÓPEZ CASTRO, J. L. (ed.) - Las ciudades fenício-púnicas en el Mediterráneo Occidental. Madrid: 459-482.

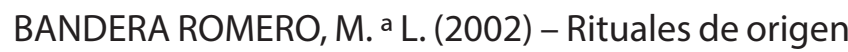
oriental entre las comunidades tartesias: el sacrificio de animales. In FERRER ALBELDA, E. (Ed.) - Ex Oriente Lux: Las Religiones Orientales Antiguas en la Peninsula Ibérica. Sevilha: 141-158.

BELÉN, M. a (2000a) - Itinerarios arqueológicos por la geografía sagrada del Extremo Occidente. In FERNÁNDEZ GÓMEZ, J. - COSTA RIBAS, B. (Coord.) Santuarios fenicio-púnicos en Iberia y su influencia en los cultos indígenas. Ibiza: 57-102.

BELÉN, M. a (2000b) - Santuarios fenicios y comercio en Tartessos. In FERNÁNDEZ URIEL, P. - GONZÁLEZ 
WAGNER, C. - LÓPEZ PARDO, F. (eds.) - Intercambio y Comercio Preclásico en el Mediterráneo. Madrid: 293312.

BELÉN, Ma (2001) - Arquitectura Religiosa Orientalizante en el Bajo Guadalquivir. In RUIZ MATA, D. - CELESTINO PÉREZ, S. (Coords.) - Arquitectura Oriental y Orientalizante en la Peninsula Iberica. Madrid: 1-16.

BERROCAL-RANGEL, L. - SILVA, A. C. (2010) - O Castro dos Ratinhos (Barragem do Alqueva, Moura). Escavações num povoado proto-histórico do Guadiana, 2004-2007. Lisboa.

CALADO, M. - MATALOTO, R. (2008) - O PostOrientalizante da Margem Direita do Regolfo do Alqueva (Alentejo Central). In JIMÉNEZ ÁVILA, J. (coord.) - Sidereum Ana I: El río Guadiana en Época Post-Orientalizante. Madrid: 185-217.

CELESTINO PÉREZ, S. (1994) - Los altares en forma de «lingote chipriota» de los santuarios de Cancho Roano. Revista de Estudios Ibéricos 1: 291-309.

CELESTINO PÉREZ, S. (1997) - Santuarios, centros comerciales y paisajes sacros. Cuadernos de Prehistoria y Arqueología Castellonenses 18: 359-389.

CELESTINO PÉREZ, S. (2001) - Los santuarios de Cancho Roano. Del Indigenismo al Orientalismo Arquitectónico. In RUIZ MATA, D. - CELESTINO PÉREZ, S. (coords.) - Arquitectura Oriental y Orientalizante en la Peninsula Iberica. Madrid: 17-56.

CELESTINO PÉREZ, S. (2008) - Los altares en forma de piel de toro de la Península Ibérica. In JUSTEL, J. J. - VITA, J. P. - ZAMORA, J. A. (eds.) - Las culturas del Próximo Oriente Antiguo y su expansión mediterránea. Madrid: 321-348.

ESCACENA CARRASCO, J. L. (2002): Dioses, toros y altares. Un templo para Baal en la antigua desembocadura del Guadalquivir. In FERRER, E. (ed.), - Ex Oriente Lux: Las religiones orientales antiguas en la Península lbérica. Sevilha: 33-76.

ESCACENA CARRASCO, J. L. - COTO SARMIENTO, M. a (2010) - Altares para la eternidad. SPAL 19: 149-185.

ESCACENA CARRASCO, J. L. - IZQUIERDO, R. (2000) Altares para Baal. ARYS 3: 11-40.

ESCALANTE AGUILAR, M. a del M. - ARANCIBIA ROMÁN, A. - CISNEROS, M. a I. - MAYORGA MAYORGA, J. (2012) - El santuario fenicio de Malaka. In GARCÍA ALFONSO, E. (ed.) - Diez Años de Arqueología Fenicia en la Provincia de Málaga (2001-2010). Málaga: 87-104. FERNÁNDEZ FLORES, A. - RODRÍGUEZ AZOGUE, A. (2007) - Tartessos desvelado. La colonización Fenicia del Suroeste peninsular y el origen y ocaso de Tartessos. Córdova.

GAMITO.T. J. (1988) - Os obeloi de bronze do Sudoeste peninsular: seu contexto e interpretação sócioideológica. In PEREIRA MENAUT. G. (ed.) - Actas del 1er. Congreso Peninsular de Historia Antigua. Santiago de Compostela, vol l: 329-339.

GOMES, F. B. (2012) - Aspectos do Sagrado na Colonização Fenícia. Lisboa.

GOMES, F. B. (2014) - Importações mediterrâneas em contextos «Pós-Orientalizantes» do Sul de Portugal (séculos VI-IV a.n.e.). Onoba 2: 27-44.

GOMES, F. B. (2015) - As oil bottles de tipo fenício do território português e o consumo de substâncias aromáticas. O Arqueólogo Português V-3: 289-310.

GÓMEZ PEÑA, Á. (2010) - Así en Oriente como en Occidente: el origen oriental de los altares taurodérmicos de la Península Ibérica. SPAL 19: 129148.

GÓMEZ PEÑA, Á. (2011) - Nuevos datos sobre altares taurodérmicos asirios y escitas y su simbología. Lucentum 30: 9-24

GÓMEZ PEÑA, Á. (2012) - Una mirada historiográfica a la identidad de los altares taurodérmicos de la Protohistoria Peninsular Ibérica. In ALDEA CELADA, J. M.-ORTEGA MARTÍNEZ, P.-PÉREZMIRANDA, I.-SOTO GARCÍA, M. a de los R. de (coords.) - Historia, Identidad y Alteridad. Actas del III Congreso Interdisciplinar de Jóvenes Historiadores. Salamanca: 735-761.

GÓMEZ PEÑA, Á. (2012-3) - Historiografía y metodología taurodérmica: nuevas consideraciones sobre su simbolismo en la protohistoria peninsular ibérica. Anales de Arqueología Cordobesa 23/24: 1134.

LÓPEZ ROSENDO, E. (2005) - El perfume en los rituales orientalizantes de la Península Ibérica. In JIMÉNEZ ÁVILA, J. - CELESTINO PÉREZ, S. (coords.) - El Período Orientalizante. Madrid, vol. I: 669-681.

MAIA, M. a (1987) - Dois larnakes da Idade do Ferro do Sul de Portugal. Veleia 2-3: 223-242.

MAIA, M. a (1988) - Neves II e a facies cultural de Neves Corvo. Arquivo de Beja 2a S. - 3: 23-42.

MAIA, M. a (2008) - Reflexões sobre os Complexos Arquitectónicos de Neves-Corvo, na região central do Baixo Alentejo, em Portugal. In JIMÉNEZ ÁVILA, J. (coord.) - Sidereum Ana I: El río Guadiana en Época Post-Orientalizante. Madrid: 353-364.

MAIA, M. - CORREA, J. A. (1985) - Inscripción en escritura tartéssica (o del SO) hallada en Neves (Castro Verde, Baixo Alentejo) y su contexto arqueológico. Habis 16: 243-274.

MAIA, M. a - MAIA, M. (1986) - Arqueologia da área mineira de Neves Corvo. Trabalhos realizados no triénio 1982-84. Castro Verde.

MAIA, M. a - MAIA, M. (1996) - Arqueologia do couto 
mineiro de Neves-Corvo. In REGO, M. (ed.) - Mineração do Baixo Alentejo. Castro Verde: 83-93.

MARÍN CEBALLOS, M. C. (2006) - De dioses, pieles y lingotes. Habis 37: 35-54.

MATALOTO, R. (2004) - Um «monte» da Idade do Ferro na Herdade da Sapatoa: ruralidade e povoamento no I milénio a.C. do Alentejo Central. Lisboa.

MATALOTO, R. (2009) - Através dos campos: arquitectura e sociedade na Idade do Ferro alto alentejana. In BELARTE, M. a C. (ed.) - L'espai domèstic i l'organització de la societat a la protohistòria de la Mediterrània occidental (ler millenni $a C$ ). Barcelona: 279-298.

MAYET, F. - SILVA, C. T. da (2000) - L'établissement phénicien d'Abul. Portugal. Paris.

PRADOS MARTíNEZ, F. (2010) - La Arquitectura sagrada: Un santuario del siglo IX A.C. In BERROCAL-
RANGEL, L. - SILVA, A. C. - O Castro dos Ratinhos (Barragem do Alqueva, Moura). Escavações num povoado proto-histórico do Guadiana, 2004-2007. Lisboa: 259-276.

RODRÍGUEZ GONZÁLEZ, E. - CELESTINO PÉREZ, S. (2017) - Las estancias de los dioses: La habitación 100 del yacimiento de Casas del Turuñuelo (Guareña, Badajoz). CuPAUAM 43: 179-194.

SÁNCHEZ, V. M. - GALINDO SAN JOSÉ, L. - JUZGADO NAVARRO, M. - MAYORGA MAYORGA, J. (2012) - EI asentamiento fenicio de La Rebanadilla a finales del siglo IX A.C.. In GARCÍA ALFONSO, E. (ed.) - Diez Años de Arqueología Fenicia en la Provincia de Málaga (2001-2010). Málaga: 25-48.

SPAGNOLI, F. (2012) - Un altare bruciaprofumi punico dalla "Casa del sacello domestico" a Mozia. Vicino Oriente 16: 71-96. 


\section{OPHIUSSA}

\section{POLÍTICA EDITORIAL}

A Ophiussa - Revista do Centro de Arqueologia da Universidade de Lisboa foi iniciada em 1996, tendo sido editado o volume 0 . A partir do volume 1 (2017) é uma edição impressa e digital da UNIARQ - Centro de Arqueologia da Universidade de Lisboa.

O principal objectivo desta revista é a publicação e divulgação de trabalhos com manifesto interesse, qualidade e rigor científico sobre temas de Pré-História e Arqueologia, sobretudo do território europeu e da bacia do Mediterrâneo.

A Ophiussa - Revista do Centro de Arqueologia da Universidade de Lisboa publicará um volume anual. A partir de 2018, os artigos submetidos serão sujeitos a um processo de avaliação por parte de revisores externos (peer review). O período de submissão de trabalhos decorrerá sempre no primeiro trimestre e a edição ocorrerá no último trimestre de cada ano.

A revista divide-se em duas secções: artigos científicos e recensões bibliográficas. Excepcionalmente poderão ser aceites textos de carácter introdutório, no âmbito de homenagens ou divulgações específicas, que não serão submetidos à avaliação por pares. Isentas desta avaliação estão também as recensões bibliográficas.

Todas as submissões serão avaliadas, em primeira instância, pela Coordenação Editorial, no que respeita ao seu conteúdo formal e à sua adequação face à política editorial e às normas de edição da revista. Os trabalhos que cumprirem estes requisitos serão posteriormente submetidos a um processo de avaliação por pares cega / blind peer review (mínimo de dois revisores). O Conselho Científico, constituído pela direcção da UNIARQ e por investigadores externos, acompanhará o processo de edição.

Esta etapa será concretizada por investigadores externos qualificados, sendo os respectivos pareceres entregues num período não superior a três meses. Os revisores procederão à avaliação de forma objectiva, tendo em vista a qualidade do conteúdo da revista; as suas críticas, sugestões e comentários serão, na medida do possível, construtivos, respeitando as capacidades intelectuais do(s) autor(es). Após a recepção dos pareceres, o(s) autor(es) tem um prazo máximo de um mês para proceder às alterações oportunas e reenviar o trabalho.

A aceitação ou recusa de artigos terá como únicos factores de ponderação a sua originalidade e qualidade científica. $O$ processo de revisão é confidencial, estando assegurado o anonimato dos avaliadores e dos autores dos trabalhos, neste último caso até à data da sua publicação.

Os trabalhos só serão aceites para publicação a partir do momento em que se conclua o processo da revisão por pares. Os textos que não forem aceites serão devolvidos aos seus autores. O conteúdo dos trabalhos é da inteira responsabilidade do(s) autor(es) e não expressa a posição ou opinião do Conselho Científico ou da Coordenação Editorial. A Revista Ophiussa segue as orientações estabelecidas pelo Commitee on Publication Ethics (COPE, Comité de Ética em Publicações): https://publicationethics.org/

O processo editorial decorrerá de forma objectiva, imparcial e anónima. Erros ou problemas detetados após a publicação serão investigados e, se comprovados, haverá lugar à publicação de correções, retratações e/ou respostas. As colaborações submetidas para publicação devem ser inéditas. As propostas de artigo não podem incluir qualquer problema de falsificação ou de plágio. Para efeito de detecção de plágio será utilizada a plataforma URKUNDU .

As ilustrações que não sejam do(s) autor(es) devem indicar a sua procedência. O Conselho Científico e a Coordenação Editorial assumem que os autores solicitaram e receberam autorização para a reprodução dessas ilustrações, e, como tal, rejeitam a responsabilidade do uso não autorizado das ilustrações e das consequências legais por infracção de direitos de propriedade intelectual.

É assumido que todos os Autores fizeram uma contribuição relevante para a pesquisa reportada e concordam com o manuscrito submetido. Os Autores devem declarar de forma clara eventuais conflitos de interesse. As colaborações submetidas que, direta ou indiretamente, tiveram o apoio económico de terceiros, devem claramente declarar essas fontes de financiamento.

Os textos propostos para publicação devem ser inéditos e não deverão ter sido submetidos a qualquer outra revista ou edição electrónica. Aceitam-se trabalhos redigidos em português, inglês, espanhol, italiano e francês.

Esta edição disponibiliza de imediato e gratuitamente a totalidade dos seus conteúdos, em acesso aberto, de forma a promover, globalmente, a circulação e intercâmbio dos resultados da investigação científica e do conhecimento.

A publicação de textos na Ophiussa - Revista do Centro de Arqueologia da Universidade de Lisboa não implica o pagamento de qualquer taxa nem dá direito a qualquer remuneração económica.

Esta publicação dispõe de uma versão impressa, a preto e branco, com uma tiragem limitada, que será distribuída gratuitamente pelas bibliotecas e instituições mais relevantes internacionalmente, e intercambiada com publicações periódicas da mesma especialidade, que serão integradas na Biblioteca da Faculdade de Letras da Universidade de Lisboa. Conta, paralelamente, com uma versão digital, a cores, disponibilizada no endereço www.ophiussa.letras.ulisboa.pt, onde se pode consultar a totalidade da edição.

Para mais informações: ophiussa@letras.ulisboa.pt 


\section{OPHIUSSA}

\section{EDITORIAL POLICY}

Ophiussa - Revista do Centro de Arqueologia da Universidade de Lisboa started in 1996, with the edition of volume 0. From 2017, this journal is a printed and digital edition of UNIARQ - Centro de Arqueologia da Universidade de Lisboa.

The main objective of this journal is the publication and dissemination of papers of interest, quality and scientific rigor concerning Prehistory and Archeology, mostly from Europe and the Mediterranean basin.

Ophiussa - Revista do Centro de Arqueologia da Universidade de Lisboa will publish an annual volume. From 2018, submitted articles will be subject to a peer-review evaluation process. The submission period will always occur in the first quarter of each year and the edition will occur in the last quarter.

The journal is divided into two sections: scientific articles and bibliographic reviews. Exceptionally, texts of an introductory nature may be accepted, in the context of specific tributes or divulgations, which will not be submitted to peer-review evaluation. Exemptions from this evaluation are also the bibliographic reviews.

All submissions will be considered, in the first instance, by the Editorial Board, regarding its formal content and adequacy in face of the editorial policy and the journal's editing standards. Papers that meet these requirements will subsequently be submitted to a blind peerreview process (minimum of two reviewers). The Scientific Council, constituted by the directors of UNIARQ and external researchers, will follow the editing process.

This stage will be carried out by qualified external researchers, and their feedback will be delivered within a period of no more than two months. The reviewers will carry out the evaluation in an objective manner, in view of the quality and content of the journal; their criticisms, suggestions and comments will be, as far as possible, constructive, respecting the intellectual abilities of the author (s). After receiving the feedback, the author(s) has a maximum period of one month to make the necessary changes and resubmit the work.

Acceptance or refusal of articles will have as sole factors of consideration their originality and scientific quality.

The review process is confidential, with the anonymity of the evaluators and authors of the works being ensured, in the latter case up to the date of its publication.

Papers will only be accepted for publication as soon as the peer review process is completed. Texts that are not accepted will be returned to their authors. The content of the works is entirely the responsibility of the author(s) and does not express the position or opinion of the Scientific Council or Editorial Board.

The Journal Ophiussa follows the guidelines established by the Commitee on Publication Ethics (COPE, the Ethics Committee Publications): https://publicationethics.org/

The editorial process will be conducted objectively, impartially and anonymously. Errors or problems detected after publication will be investigated and, if proven, corrections, retractions and / or responses will be published. Contributions submitted for publication must be unpublished. Article submissions can not include any problem of forgery or plagiarism. In order to detect plagiarism, the URKUNDU platform will be used.

Illustrations that are not from the author(s) must indicate their origin. The Scientific Council and Editorial Board assume that the authors have requested and received permission to reproduce these illustrations and, as such, reject the responsibility for the unauthorized use of the illustrations and legal consequences for infringement of intellectual property rights.

It is assumed that all Authors have made a relevant contribution to the reported research and agree with the manuscript submitted. Authors must clearly state any conflicts of interest. Collaborations submitted that directly or indirectly had the financial support of third parties must clearly state these sources of funding.

Texts proposed for publication must be unpublished and should not have been submitted to any other journal or electronic edition. Works written in Portuguese, English, Spanish, Italian and French are accepted.

The publication of texts in Ophiussa - Revista do Centro de Arqueologia da Universidade de Lisboa does not imply the payment of any fee nor does it entitle to any economic remuneration.

This edition immediately and freely provides all of its content, in open access, in order to promote global circulation and exchange of scientific research and knowledge.

This publication has a limited printed edition in black and white, which will be distributed free of charge by the most relevant international libraries and institutions, and exchanged with periodicals of the same specialty, which will be integrated in the Library of Faculdade de Letras of Universidade de Lisboa. It also has a digital version, in color, available at address http://ophiussa.letras.ulisboa.pt, where one can consult the entire edition.

For more information contact: ophiussa@letras.ulisboa.pt 


\section{ÍNDICE}

CRISTINA GAMEIRO - A tecnologia lítica do fim do Tardiglaciar no centro de Portugal: o exemplo do Abrigo 1 de Vale de Covões (Soure)

JUAN ANTONIO CÁMARA SERRANO - FERNANDO MOLINA GONZÁLEZ - CRISTÓBAL PÉREZ BAREAS LILIANA SPANEDDA - Una nueva lectura de las fortificaciones calcolíticas del Cerro de la Virgen (Orce, Granada, España)

THOMAS TEWS - A quadratura do círculo: sobre a questão da escolha de planta na arquitectura doméstica, no exemplo da Pré-História Recente e Proto-História na Estremadura Portuguesa .. 39 ÍRIS DA COSTA DIAS - A ocupação da Serra do Socorro (Mafra, Torres Vedras) durante o Bronze Final: a colecção de Gustavo Marques

FRANCISCO JOSÉ GARCÍA FERNÁNDEZ - FERNANDO AMORES CARREDANO - ROCIOO IZQUIERDO DE MONTES - ANA MARÍA JIMÉNEZ FLORES - Dos enterramientos singulares de la necrópolis de la Cruz del Negro (Carmona, Sevilla)

FRANCISCO B. GOMES - Equipamentos de culto nos santuários da Idade do Ferro do Sul de Portugal: os altares

ANA SOFIA ANTUNES - Fornos / silos aéreos da arquitectura sidérica peninsular: a propósito de uns "fundos de cabana" e de umas estruturas circulares da Azougada

ANTONIO M. SÁEZ ROMERO - Pucheros y fogones. Aproximación a la evolución de la producción de «cerámicas de cocina» púnicas y tardopúnicas en Gadir .....

MARIA JOSÉ DE ALMEIDA - Contributo para a normalização do registo de informação arqueológica a partir do estudo da via Emerita-Olisipo por Ebora

ALEXANDRA NEPOMUCENO - Fragmentos do Oriente em Leite Vasconcelos

DANIEL CARVALHO - A História da Arqueologia no novo milénio: dimensões, métodos e perspectivas para o caso português

RECENSÕES BIBLIOGRÁFICAS (textos de Juan Álvarez García, Francisco B. Gomes e Elisa de Sousa)

JEAN GUILAINE. DOUTOR HONORIS CAUSA PELA UNIVERSIDADE DE LISBOA (textos de Mariana Diniz, Victor S. Gonçalves e Jean Guilaine)

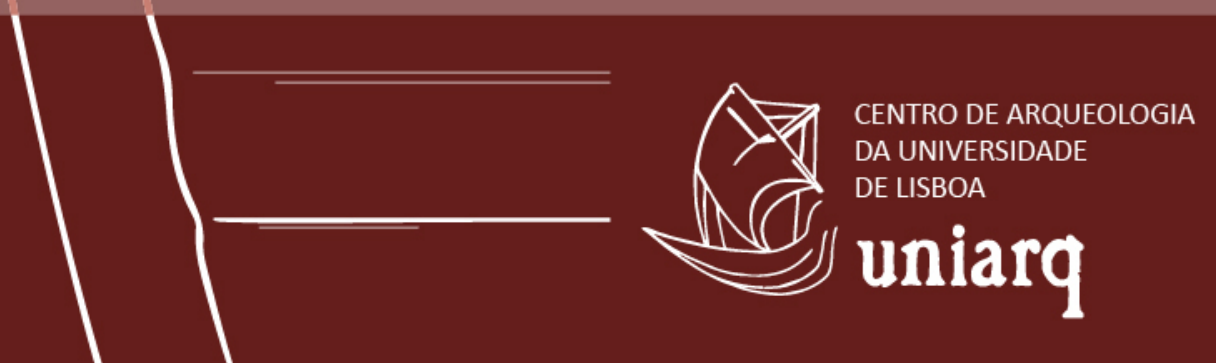

